Classification

Physics Abstracts

$47.55 \mathrm{M}$

\title{
Behaviour of oil ganglia displaced by a surfactant solution in a porous medium
}

\author{
J. C. Moulu \\ Institut Français du Pétrole, B.P. 311, 92506 Rueil-Malmaison, France
}

(Reçu le 7 septembre 1984, révisé le 26 octobre, accepté le 4 décembre 1984)

\begin{abstract}
Résumé. - La vitesse de globules d'huile entraînés dans un milieu poreux par une solution de tensioactif est mesurée. Un modèle analytique qui relie la vitesse des globules aux paramètres de l'écoulement de la solution de tensio-actif est établi. L'expérience vérifie ce modèle et met en évidence les différents régimes du mouvement des globules définis par l'importance relative des forces de viscosité et des forces capillaires.

Abstract. - The velocity of oil ganglia displaced by a surfactant solution in a porous medium is measured. With the help of an analytical model, a relationship between the velocity of the blobs and the parameters of the surfactant flood is determined. This model is in good agreement with experimental results. According to the relative importance of viscous and capillary forces, the different regimes of the ganglion motion are identified.
\end{abstract}

1. - A number of authors have used the concept of competition between viscous and capillary forces in describing the displacement of a residual oil phase by water injection in a porous medium $[1,2]$. These studies have demonstrated that higher viscous/capillary forces ratios are needed to recover residual phases than are commonly encountered in ordinary oil production processes by water.

The purpose of tertiary oil recovery processes is to mobilize a significant part of this residual oil by drastically reducing the interfacial tension with a surfactant injection.

For a non-wetting immobile oil phase, such as that shown in figure 1 for a single blob, a simple force balance $[3,4]$ demonstrates that the oil mass will be displaced if the applied pressure exceeds the net restraining capillary pressure.

An aqueous surfactant solution injected in a linear porous medium at a mean superficial velocity $V_{\mathrm{w}}$ creates a pressure gradient $\mathrm{d} P / \mathrm{d} l$ given by Darcy's law :

$$
\frac{\mathrm{d} P}{\mathrm{~d} l}=\frac{\mu_{\mathrm{w}} V_{\mathrm{w}} \phi}{k}
$$

where $\mu_{\mathrm{w}}$ is the viscosity of the solution.

The porous medium is characterized by its permeability $k$ and its porosity $\phi$. Then the pressure difference applied to an oil blob trapped in the porous medium is :

$$
\Delta P=\frac{\mu_{\mathrm{w}} V_{\mathrm{w}} \phi}{k} \cdot L
$$




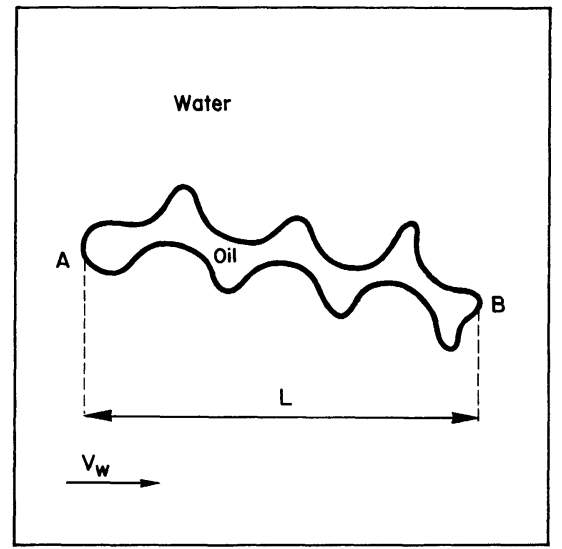

Fig. 1. - Schematic view of an oil blob in a porous medium.

$L$ being the projected length of the blob along the direction of the flow. The blob remains stranded if this pressure difference is smaller than the difference $\Delta P_{\mathrm{c}}$ between the capillary pressures measured at the two points $\mathrm{A}$ and $\mathrm{B}$ with :

$$
\Delta P_{\mathrm{c}}=2 \sigma\left(\frac{1}{r_{\mathrm{B}}}-\frac{1}{r_{\mathrm{A}}}\right)
$$

where $\sigma$ is the interfacial tension between the oleic phase of the blob and the surfactant solution, $r_{\mathrm{B}}$ the pore throat radius where the blob is trapped, and $r_{\mathrm{A}}$ the radius of the pore at the trailing edge of the blob.

Assuming that $r_{\mathrm{A}} \gg r_{\mathrm{B}}=r_{\mathrm{n}}, r_{\mathrm{n}}$ being the radius of the neck $\mathrm{B}$, we can write :

$$
\Delta P_{\mathrm{c}} \sim \frac{2 \sigma}{r_{\mathrm{n}}} .
$$

If the difference of pressure created by the flood of the aqueous solution becomes higher than the difference of capillary pressures, the blob is mobilized with a velocity $V_{\mathrm{o}}$ given by Poiseuille's law in a capillary of radius $r$ and :

$$
\Delta P-\Delta P_{\mathrm{c}}=\frac{8 \mu_{\mathrm{o}} V_{\mathrm{o}}}{r^{2}} \cdot L
$$

or

$$
\frac{\mu_{\mathrm{w}} V_{\mathrm{w}} \phi}{k} \cdot L-\frac{2 \sigma}{r_{\mathrm{n}}}=\frac{8 \mu_{\mathrm{o}} V_{\mathrm{o}}}{r^{2}} \cdot L,
$$

where $\mu_{\mathrm{o}}$ is the viscosity of the oil phase and

$$
\frac{\mu_{\mathrm{w}} V_{\mathrm{w}} \phi}{\sigma}-\frac{2 k}{L r_{\mathrm{n}}}=\frac{8 k}{r^{2} \phi} \cdot \frac{\mu_{\mathrm{o}} V_{\mathrm{o}} \phi}{\sigma} .
$$

Let us consider the capillary numbers : $N_{\mathrm{cw}}=\mu_{\mathrm{w}} V_{\mathrm{w}} \phi / \sigma$ for the surfactant solution and $N_{\text {co }}=\mu_{\mathrm{o}} V_{\mathrm{o}} \phi / \sigma$ for the oil phase, which represent the ratio of the viscosity over the capillary 
forces; we have :

$$
N_{\mathrm{cw}}=\frac{8 k}{r^{2} \phi} \cdot N_{\mathrm{co}}+\frac{2 k}{L r_{\mathrm{n}}} .
$$

It is well-known (see for example [5]) that a bundle of cylindrical capillaries of radius $r^{*}$ has a permeability $k$ and a porosity $\phi$ such that :

$$
r^{*}=\sqrt{\frac{8 k}{\phi}} .
$$

A real porous medium has a pore radius $r$ like that used in relation (3) proportional to $r^{*}$ and thus :

$$
r=a \sqrt{\frac{8 k}{\phi}}
$$

with « $a$ » depending on the geometry and topology of the porous medium.

Finally relation (3) can be written :

$$
N_{\mathrm{cw}}=\frac{N_{\mathrm{co}}}{a^{2}}+\frac{2 k}{L r_{\mathrm{n}}} .
$$

\section{Experimental.}

The fluids used in the experiments are aqueous solutions containing $2 \%$ wt surfactant(TRS 10-80) and alcohol (n-pentanol) with different salt concentrations $(1.5$ to $9 \mathrm{~g} / 1 \mathrm{NaCl})$. The parameters of these solutions are measured at the experimental temperature, and more precisely :

- the interfacial tension between oil and aqueous solutions is time dependent because these formulations are non-equilibrated and the partitioning of alcohol and surfactant between oil and water phases needs time to establish. The values of interfacial tension are found to be between $2 \times 10^{-1}$ and $4 \times 10^{-3} \mathrm{mN} / \mathrm{m}$, depending on the different formulation salinities,

- the viscosities are evaluated by injecting the solutions in the different porous media used for the experiments with a large range of mean superficial velocities corresponding to the displacement of the blob. Among the surfactant solutions, some show a non-Newtonian rheological behaviour (Fig. 2) and a very important dependence of the shear rate on the viscosity values.

The porous media considered in this study are :

- on the one hand, micromodels made with a single layer of glass beads of diameter $750 \mu \mathrm{m}$ or $2 \mathrm{~mm}$ and corresponding porosities 33 and $44 \%$,

- on the other hand, cylindrical cores from pure Fontainebleau sandstone having permeabilities 0.350 and $1.3 \mu^{2}$ and corresponding porosities 11 and $18 \%$.

With glass-bead models, the experiments consist of introducing a small quantity of oil in the porous medium by way of a hole drilled through a glass side of this model, as has been explained elsewhere $[6,7]$.

Measurements are taken between the mobilization of the ganglion by the surfactant flood, from an initial position until its stranding. The travelled path varies from $1 \mathrm{~cm}$ to $20 \mathrm{~cm}$ and the time of travel from $3 \mathrm{~min}$ to $30 \mathrm{~min}$. The behaviour of each blob and of daughter-blobs after break-up are followed by a camera-video recorder and their velocity calculated at $1 \mathrm{~cm}$ intervals.

With sandstones, it is only possible to evaluate the resident time of small quantities of oil between their injection and their breakthrough in the effluent; two permeabilities and different 


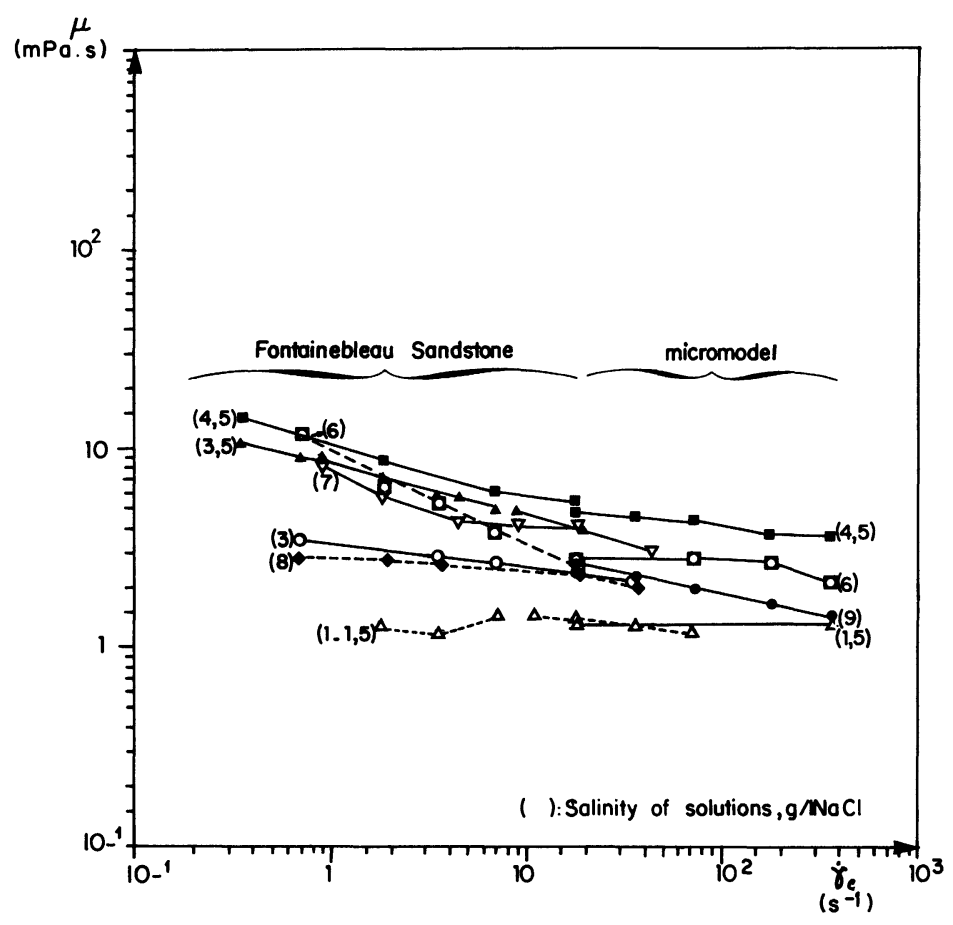

Fig. 2. - Rheological behaviour of the surfactant solutions.

lengths of cores are used. After each experiment with a given aqueous solution and a constant injection rate, the porous media are washed with isopropyl alcohol, then heated and dried with nitrogen before being saturated with water and flooded by another surfactant solution.

\section{Results.}

From the measurement of time and length of travel for different blobs, mobilized by different aqueous solutions of surfactant, hence various values of interfacial tension and viscosity, it has been observed that, in glass bead models, the velocity of a blob is independent of its length, although this blob usually breaks up many times in the porous medium.

It is assumed that blobs exhibit the same behaviour in sandstone cores. From these measurements and the knowledge of :

- interfacial tension, the value of which is taken at a time corresponding to the mean travel time in the porous medium,

- viscosity, measured at the shear rate corresponding to given flow rate,

it is possible to calculate the capillary numbers $N_{\mathrm{cw}}$ and $N_{\mathrm{co}}$. The relation $N_{\mathrm{cw}} v \boldsymbol{v} . N_{\mathrm{co}}$ is plotted as shown in figure 3, which represents the points for different solutions, the capillary number of which is increased by the increase of flow rate.

Different motion regimes of the blobs can be defined :

- if $N_{\mathrm{cw}}$ values are low, the blobs remain stranded by capillary forces and $N_{\mathrm{co}}=0$,

- if $N_{\mathrm{cw}}$ is higher than a level given by $2 k / L r_{\mathrm{n}}$ (see Eq. (4)), the oil blobs are mobilized and have for a given interfacial tension, velocities increasing with $N_{\mathrm{cw}}$. The mobilization level of the blobs can be roughly evaluated : in the case of the $2 \mathrm{~mm}$ glass-bead micromodel (Fig. 3) with 


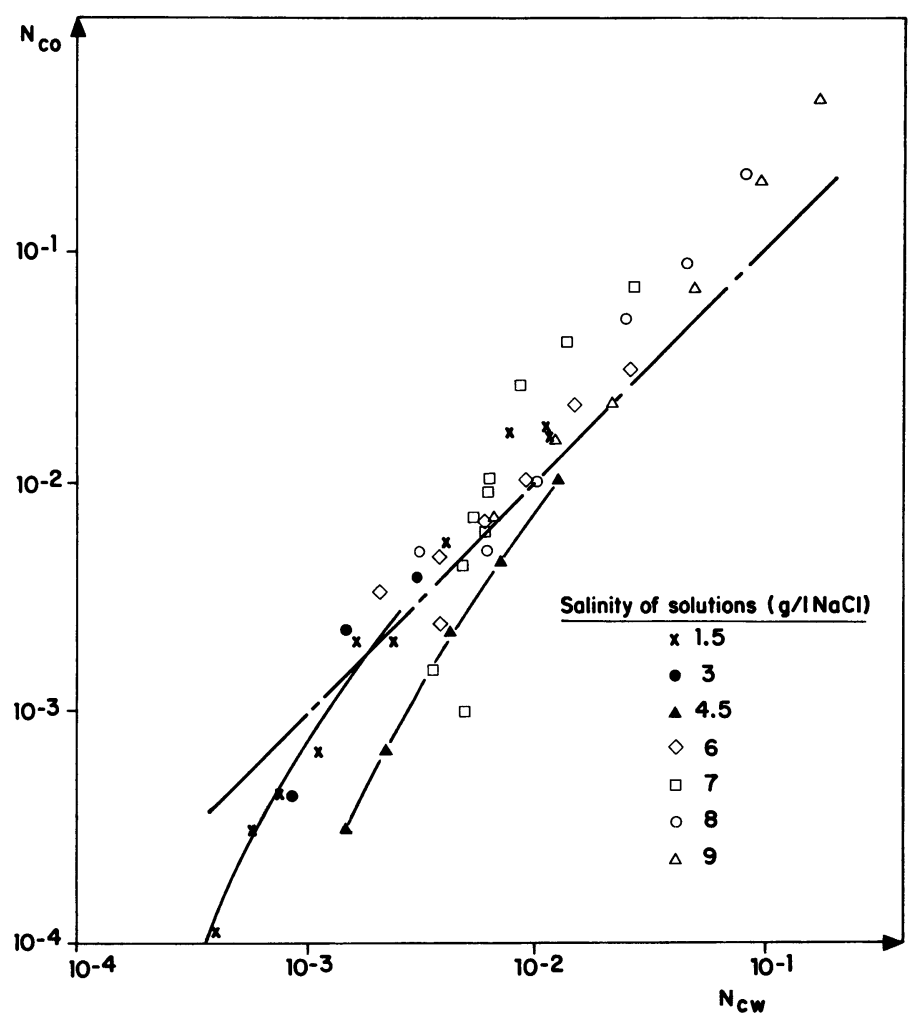

Fig. 3. - Dependence between the capillary numbers for water and for oil. Glass beads $=2 \mathrm{~mm}$.

a $700 \mu \mathrm{m}^{2}$ permeability and a neck radius $r_{\mathrm{n}}=250 \mu \mathrm{m}$, the size of the blobs having been measured during their displacement $\left(L \sim 10^{-2} \mathrm{~m}\right)$, then :

$$
\frac{2 k}{L r_{\mathrm{n}}} \sim 5.6 \times 10^{-4}
$$

This value, although a bit high [8], is well representative of the minimum for $N_{\mathrm{cw}}$ over which the mobilization of blobs has been really observed during the experiments,

- if $N_{\mathrm{cw}}$ is high enough, the term $2 k / L r_{\mathrm{n}}$ can be neglected and the relation (4) becomes :

$$
N_{\mathrm{cw}}=\frac{N_{\mathrm{co}}}{a^{2}}
$$

and therefore, the corresponding log-log representation is a straight line with a slope of unity which is at the distance $a^{2}$ of the line $N_{\mathrm{cw}}=N_{\mathrm{co}}$. We find the same result for the different porous media studied and if we only consider the linear part of their curve (Fig. 4), we can see that :

- the points for each medium are found to follow a linear trend with slope unity, and the relation $N_{\mathrm{cw}}=N_{\mathrm{co}} / a^{2}$ is well verified in the range of high capillary number values,

- the distance between this straight line and the first bisectrix gives the constant $a$ value 


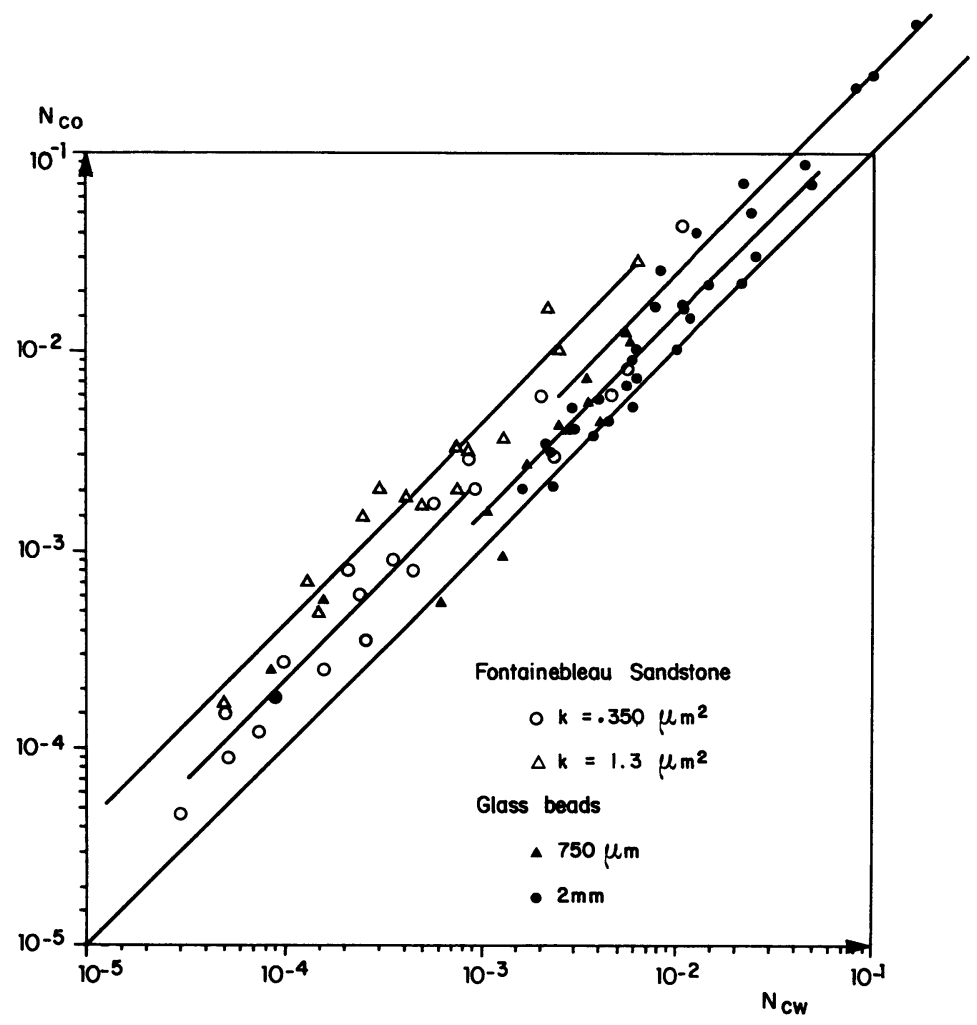

Fig. 4. - Correlation between $N_{\mathrm{cw}}$ and $N_{\mathrm{co}}$ for low capillary forces.

for each medium. We can estimate :

$$
\begin{aligned}
& a \sim 1.4 \text { for Fontainebleau sandstone } \\
& a \sim 1.2 \text { for the micromodels } .
\end{aligned}
$$

Other works [9] show values of $a$ about 1.6 for a Fontainebleau sandstone and about 1 for unconsolidated porous media being three dimensional and therefore quite different from ours which are made of a single layer of glass beads.

In other respects, it can be observed that relation (5) is equivalent to :

$$
\mu_{\mathrm{w}} V_{\mathrm{w}}=\frac{\mu_{\mathrm{o}} V_{\mathrm{o}}}{a^{2}}
$$

and shows that in this linear part of the relation between $N_{\mathrm{cw}}$ and $N_{\mathrm{co}}$, the $a$ value is not dependent on interfacial tension values which are roughly estimated from kinetic curves obtained out of porous media.

These first results show that, in a porous medium, the behaviour of the wetting phase, here an aqueous surfactant solution, can be represented by Darcy's law including the permeability $k$ of the medium; the non-wetting phase, here a unique oil blob, is considered moving in a pore having a mean radius $r$. A relation established between $k$ and $r$ can be used for investigating the porous medium and for describing it with a value of pore radius more realistic than $\sqrt{8 k / \phi}$. 
Besides, other works now in progress in our laboratories show the importance of blob velocity, compared with injection rate, on oil bank formation which is a general condition of success for an enhanced oil recovery process.

\section{References}

[1] Foster, W. R., J. Pet. Technol. 25 (1973).

[2] Abrams, A., Soc. Pet. Eng. J. 15 (1975).

[3] Melrose, J. C. and Brandner, C. F., Can. J. Pet. Technol. 13, no 4 (1974).

[4] Stegemeier, G. L., Soc. Pet. Eng. 4754, Presented at Improved Oil Recovery Symposium, Tulsa. Oklahoma, April 1974.

[5] Scheidegger, A. E., Pet. Eng. B 25 (1953) 121.

[6] Payatakes, A. C., Annu. Rev. Fluid Mech. 14 (1982) 365.

[7] RapIN, S., M.S. Thesis. Univ. Houston (1980).

[8] Moulu, J. C., Revue de l'Institut Français du Pétrole 39, no 1 (1984).

[9] Chauveteau, G., European Symposium on Enhanced Oil Recovery Bournemouth, England (1981) p. 197. 